\title{
Development of retinoic acid syndrome during leukopenia
}

\author{
Katsuki Sugiyama $\cdot$ Noriko Usui $\cdot$ Nobuaki Dobashi $\cdot$ \\ Shingo Yano $\cdot$ Yutaka Takei $\cdot$ Shinobu Takahara \\ Takeshi Saito $\cdot$ Keisuke Aiba
}

Received: 20 August 2011/Accepted: 8 November 2011/Published online: 20 December 2011

(C) The Japan Society of Clinical Oncology 2011

\begin{abstract}
We present a case involving a 53-year-old male with acute promyelocytic leukemia who developed retinoic acid syndrome (RAS) during leukopenia. He received alltrans retinoic acid (ATRA) therapy at a dose of $90 \mathrm{mg} /$ day $\left(45 \mathrm{mg} / \mathrm{m}^{2}\right.$ ). On day 17 , his fever increased to $38.1^{\circ} \mathrm{C}$. As pain developed in his left ankle on day 19, ATRA was discontinued on day 20 because of the suspicion of an adverse reaction to the therapy such as RAS. On day 28 , his fever increased markedly, and respiratory distress and bilateral pleural effusion developed. Although his maximum leukocyte count during the course of the condition was low $(3,800 / \mu \mathrm{l})$, steroid pulse therapy was started on day 32. Thereafter, these symptoms improved dramatically. Based on the clinical manifestations observed and the patient's complete response to steroid pulse therapy, RAS was considered to have developed in the present case. RAS is associated with the development of hyperleukocytosis in most patients, but a few patients with normal leukocyte counts have developed the syndrome. As RAS is a fatal complication of ATRA therapy, early diagnosis and treatment are very important. The present case emphasizes the view that when administering ATRA, RAS should be kept in mind at all times.
\end{abstract}

Keywords Acute promyelocytic leukemia $\cdot$ All-trans retinoic acid $\cdot$ Retinoic acid syndrome $\cdot$ Leukopenia

K. Sugiyama $(\bowtie) \cdot$ N. Usui · N. Dobashi $\cdot$ S. Yano ·

Y. Takei $\cdot$ S. Takahara $\cdot$ T. Saito $\cdot$ K. Aiba

Division of Clinical Oncology and Hematology, Department of Internal Medicine, The Jikei University School of Medicine, 3-25-8 Nishi-shinbashi, Minato-ku, Tokyo 105-8461, Japan e-mail: katsuki@jikei.ac.jp

\section{Introduction}

All-trans retinoic acid (ATRA) significantly improves the outcome of patients with acute promyelocytic leukemia (APL) [1]. Retinoic acid syndrome (RAS), which is the most serious complication of ATRA therapy, presents with clinical symptoms such as dyspnea, fever, weight gain, hypotension, acute renal failure, pulmonary infiltrates, and pleuropericardial effusion [2]. It is associated with the development of hyperleukocytosis in most patients, but a few patients with normal leukocyte counts have developed the syndrome. Here, we report on a patient with APL who was treated using ATRA and developed RAS during leukopenia.

\section{Case report}

A 53-year-old male was admitted to our hospital because of a bleeding tendency and pancytopenia in October 2002. His laboratory data revealed a white blood cell (WBC) count of $800 / \mu \mathrm{l}$ (4\% blasts and $14 \%$ promyelocytes), a hemoglobin level of $4.3 \mathrm{~g} / \mathrm{dl}$, a platelet count of $9,000 / \mu \mathrm{l}$, a fibrinogen level of $137 \mathrm{mg} / \mathrm{dl}$, and a fibrin degradation product level of $99 \mu \mathrm{g} / \mathrm{ml}$ (disseminated intravascular coagulation was noted). Bone marrow aspiration revealed hypercellularity with $7.2 \%$ blasts and $86.0 \%$ promyelocytes. Immunophenotypic analysis of the leukemic cells revealed the expression of CD13 (80.1\%), CD33 (95.1\%), and CD34 (25.1\%). Cytogenetic analysis revealed a karyotype of 46, $\mathrm{XY}, \mathrm{t}(15 ; 17)(\mathrm{q} 22 ; \mathrm{q} 21)[20 / 20]$. The diagnosis of APL was made based on the above findings. The clinical course of the patient is shown in Fig. 1. Induction therapy with ATRA was started at a dose of $90 \mathrm{mg} /$ day $\left(45 \mathrm{mg} / \mathrm{m}^{2}\right)$. On day 17 , his fever increased to $38.1^{\circ} \mathrm{C}$, and empiric 


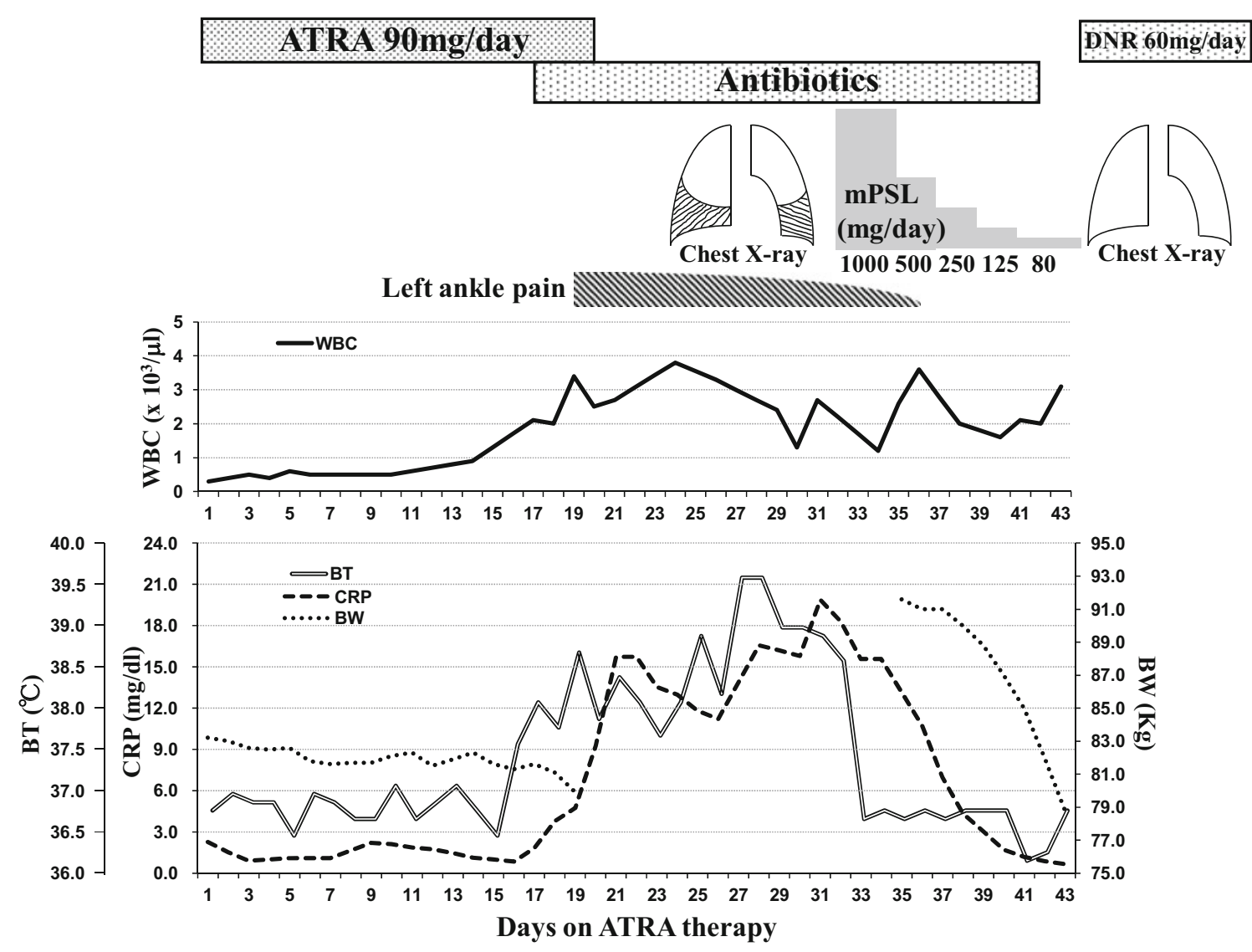

Fig. 1 Clinical course of the patient. ATRA all-trans retinoic acid, DNR daunorubicin, $m P S L$ methylprednisolone, WBC white blood cell, $B T$ body temperature, $C R P$ C-reactive protein, $B W$ body weight

antibiotic therapy was started. As he developed pain in his left ankle on day 19, ATRA was discontinued on day 20 because of the possibility that it had been caused by an adverse reaction to the therapy. On day 28 , his fever increased markedly $\left(39^{\circ} \mathrm{C}\right)$, and hypoxemia $\left(\mathrm{O}_{2}\right.$ saturation $94 \%$ in room air) developed. A chest X-ray and computed tomography revealed pleural effusion bilaterally, but findings indicative of pneumonia were seen only in the lower left lung field, suggesting that RAS had developed (Fig. 2). At that time, since the existence of an infection could not be completely neglected, the administration of the steroid was interrupted. However, despite the administration of antibiotics, the patient's respiratory distress became worse thereafter, and his pleural effusion tended to increase. Although his WBC counts were consistently low, with a maximum count of $3,400 / \mu 1$ during ATRA administration and a maximum count of $3,800 / \mu \mathrm{l}$ after ATRA discontinuation, steroid pulse therapy was finally started on day 32 because of a suspicion of RAS having developed. Thereafter, his fever, hypoxemia, pleural effusion, and left ankle pain improved dramatically. The patient's body weight could not be measured when the pain was present in his left ankle, but it became possible to start measuring it again on day 35 because of the improvement of his condition. At that time, he weighed $91.6 \mathrm{~kg}$, which represented an increase of about $10 \mathrm{~kg}$ from the last measurement. On day 42 , his body weight had decreased to the value measured at the time of the initiation of ATRA therapy. Thereafter, since he had not achieved complete remission, he received $60 \mathrm{mg} /$ day of daunorubicin for 7 days and entered complete remission. Subsequently, he received three courses of consolidation chemotherapy without ATRA and has now been in complete remission for 8 years.

\section{Discussion}

RAS is associated with the development of hyperleukocytosis in most patients, but a few patients with normal leukocyte counts have developed the syndrome. The pathogenesis of the syndrome is still not completely understood. However, several possible mediators have been identified, including hematopoietic growth factors and cell adhesion molecules on APL cells [3]. In the reports by Frankel et al. [2] and Tallman et al. [4], the median WBC 
Fig. 2 Chest X-ray

(a) and computed tomography

(b) revealed pleural effusion

bilaterally. Findings indicative of pneumonia were only seen in the lower left lung field
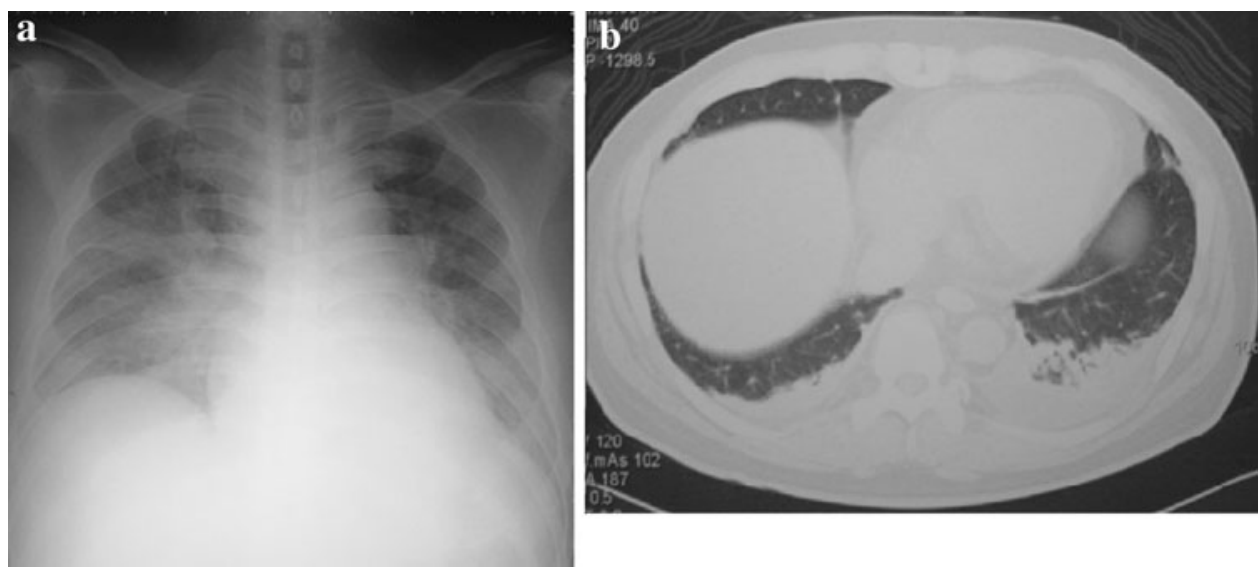

count at the time of RAS onset was $36,400 / \mu$ l (range: $6,300-82,300 / \mu \mathrm{l}$ ) and $31,000 / \mu \mathrm{l}$ (range: 6,800-72,000/ $\mu \mathrm{l}$ ), respectively. In the report by Vahdat et al. [5], the median WBC count was $34,100 / \mu 1$, and one reported case had a WBC count of no more than $5,000 / \mu$ l (no further information provided). It has also been reported that a high WBC count at diagnosis or rapidly increasing WBC counts during ATRA therapy could be predictive factors of RAS [6]. Recently, Montesinos et al. identified a WBC count greater than $5,000 / \mu 1$ at diagnosis as an independent factor predictive of the development of severe RAS. They classified patients with four or more symptoms of RAS as having severe RAS. However, they did not find any relationship between the rate of WBC increase and the development of RAS [7].

Based on the clinical manifestations observed and the patient's complete response to steroid pulse therapy, RAS was considered to have developed in the present case, which was classified as severe RAS, according to the criteria described in the report by Montesinos et al. However, the maximum WBC count during the course of this patient's condition was $3,800 / \mu \mathrm{l}$ (WBC count at diagnosis: $800 / \mu \mathrm{l})$, and, as far as we know, our patient had the lowest WBC count among all the previously reported cases. In the present case, there was a possibility that discontinuing ATRA before the appearance of leucocytosis contributed to the absence of leucocytosis. Another possibility was that differentiated APL cells were present extravascularly. Ohno et al. reported that at least ten times more neutrophils are present extravascularly than intravascularly. Therefore, even if a patient shows no increase in their number of peripheral leukocytes at the presentation of RAS, this does not exclude an association between an increase in the number of neutrophils and this syndrome because leukocytosis eventually appears, even in cases without leukocytosis at the presentation of RAS [8]. Moreover, Botton et al. [9] reported that the early addition of chemotherapy to ATRA in patients with newly diagnosed APL and low
WBC counts (below 5,000/ $\mu \mathrm{l}$ ) significantly reduced the incidence of RAS. Their report also suggested that a large number of neutrophils are located extravascularly.

In general, RAS is diagnosed based on clinical symptoms such as dyspnea, fever, weight gain, hypotension, acute renal failure, pulmonary infiltrates, and pleuropericardial effusion [2]. However, during induction therapy it is not uncommon for severe complications such as fever, pneumonia, and septicemia to develop and aggravate the patient's general status. Therefore, diagnosing RAS is not always easy when the patient's WBC count is low. In the present case, although fever and respiratory distress were seen, the patient's WBC counts were consistently low. In addition, body weight could not be measured throughout the study due to the patient suffering from ankle pain. As RAS is a fatal complication of ATRA therapy, early diagnosis and treatment are very important. The present case emphasizes the view that when administering ATRA, RAS should be kept in mind at all times.

\section{Conflict of interest None.}

\section{References}

1. Tallman MS, Nabhan C, Feusner JH et al (2002) Acute promyelocytic leukemia: evolving therapeutic strategies. Blood 99: 759-767

2. Frankel SR, Eardley A, Lauwers G et al (1992) The 'retinoic acid syndrome' in acute promyelocytic leukemia. Ann Intern Med 117:292-296

3. Fenaux P, Chomienne C, Degos L (2001) All-trans retinoic acid and chemotherapy in the treatment of acute promyelocytic leukemia. Semin Hematol 38:13-25

4. Tallman MS, Andersen JW, Schiffer CA et al (2000) Clinical description of 44 patients with acute promyelocytic leukemia who developed the retinoic acid syndrome. Blood 95:90-95

5. Vahdat L, Maslak P, Miller WH Jr et al (1994) Early mortality and the retinoic acid syndrome in acute promyelocytic leukemia: impact of leukocytosis, low-dose chemotherapy, PML/RAR- $\alpha$ isoform, and CD13 expression in patients treated with all-trans retinoic acid. Blood 84:3843-3849 
6. Fenaux P, Botton SD (1998) Retinoic acid syndrome, recognition, prevention and management. Drug Saf 18:273-279

7. Montesinos P, Bergua JM, Vellenga E et al (2009) Differentiation syndrome in patients with acute promyelocytic leukemia treated with all-trans retinoic acid and anthracycline chemotherapy: characteristics, outcome, and prognostic factors. Blood 113:775-783

8. Ohno R, Asou N, Ohnishi K (2003) Treatment of acute promyelocytic leukemia: strategy toward further increase of cure rate. Leukemia 17:1454-1463
9. De Botton S, Chevret S, Coiteux V et al (2003) Early onset of chemotherapy can reduce the incidence of ATRA syndrome in newly diagnosed acute promyelocytic leukemia (APL) with low white blood cell counts: results from APL 93trial. Leukemia $17: 339-342$ 